\title{
El contagio de robos de vehículos y sustracciones de objetos en vehículos: Aplicaciones de técnicas epidemiológicas.
}

\author{
Lucía Summers, Shane D. Johnson \& Ken Pease \\ UCL Jill Dando Institute of Crime Science. UK \\ e-mail: l.summers@ucl.ac.uk
}

\section{RESUMEN}

La aplicación de técnicas epidemiológicas al estudio del robo de domicilios ha demostrado que éstos se aglomeran espacio-temporalmente y de forma predecible (Johnson y Bowers, 2004; Johnson, Birks et al., 2007; Townsley et al., 2003). Es decir, una vez que un delito ocurre, es más probable que otro ocurra a poca distancia y poco después del original; en términos epidemiológicos, el riesgo de este tipo de victimización es altamente contagioso. En este estudio, análisis de denuncias de robos de vehículos y sustracciones de objetos en vehículos en los condados de Derbyshire y Dorset (Reino Unido) a lo largo de un año demostraron que estas pautas también son aplicables a la sustracción de objetos en vehículos pero no al robo de vehículos. El trazado de mapas predictivos basados en estos conocimientos podría ser útil y contribuir a la prevención del delito y la detección y detención de delincuentes.

Palabras clave: Robo de vehículo; sustracción de objetos en vehículo; modelos espacio-temporales; mapas predictivos; técnicas epidemiológicas.

\section{ABSTRACT}

The application of epidemiological techniques to the study of burglary has demonstrated that these cluster in space and time (Johnson \& Bowers, 2004; Johnson, Birks et al., 2007; Townsley et al., 2003). In other words, when an offence occurs, another is likely to occur nearby and soon after; in epidemiological terms, the risk of this type of victimisation is highly communicable. In this study, analyses of one year's worth of theft from and theft of motor vehicle police data for Derbyshire and Dorset (UK) demonstrated that these patterns are also found for theft from motor vehicle but not for theft of motor vehicle. Prospective mapping based on these findings could be useful in improving crime reduction and offender detection.

Keywords: Theft from motor vehicle; theft of motor vehicle; spatio-temporal models; predictive mapping; epidemiological techniques.

Revista Española de Investigación Criminológica 


\section{Introducción}

La predicción de dónde y cuándo es más probable que se cometan delitos en un futuro facilitaría la prevención de los mismos y la aprehensión de los delincuentes que los cometen. Hasta ahora, se ha demostrado que:

- el delito no se distribuye de forma aleatoria, ni espacial ni temporalmente (ver Chainey y Ratcliffe, 2005);

- el mejor predictor de la victimización es la victimización previa (e.g. Pease, 1998); y

- la victimización futura de una ubicación se puede predecir no sólo en base a la victimización previa propia, sino también en base a la victimización de ubicaciones próximas a ésta (e.g. Townsley et al., 2003; Johnson, Bernasco et al., 2007).

Estos conocimientos se han utilizado para producir mapas de 'puntos calientes', que se utilizan en muchos cuerpos policiales para organizar el patrullaje preventivo. No obstante, estos mapas son históricos por lo que su valor predictivo es limitado (e.g. Bowers et al., 2004). Como lo expresan Bowers et al. (2004: 641), 'aunque la información que tenemos se refiere al pasado, es el futuro lo que tenemos que saber'.

Técnicas desarrolladas en el campo de la epidemiología para estudiar las pautas espacio-temporales de las enfermedades contagiosas se pueden aplicar al estudio del delito para realizar predicciones y así facilitar la producción de mapas predictivos (en ves de mapas históricos).

Uno de los pioneros de estas técnicas fue Knox, que en 1964 publicó un artículo en el que aplicaba estos métodos el estudio de la leucemia infantil, ya que en aquellos tiempos la etiología de esta enfermedad era poco conocida. Knox (1964) desarrolló un método por el cual se calculan las distancias espacio-temporales de todos los posibles pares de incidencias (para un total de $n$ incidencias, habría $(n(n-1)) / 2$ ! parejas de incidencias o combinaciones). Después de categorizar las distancias geográficas y temporales en intervalos fijos, se crea una tabla de contingencia para contar el número de combinaciones en cada categoría. Por ejemplo, la casilla $\mathrm{n}_{11}$ representaría el número 
de pares de incidencias que ocurren a menos de 100 metros y en menos de 14 días (tabla1 ). El significado estadístico de estas frecuencias se puede entonces determinar al comparar éstas con las frecuencias esperadas, calculadas sobre los totales (test de chi cuadrado). El problema con este método es que las incidencias no son independientes entre ellas, lo cual es una condición de este test.

\begin{tabular}{|c|c|c|c|c|c|c|}
\hline & & \multicolumn{5}{|c|}{ Distancia Temporal } \\
\hline & & 14 días & 28 días & 42 días & $\ldots$ & $14 \mathrm{n}$ días \\
\hline \multirow{5}{*}{$\begin{array}{l}\text { Distancia } \\
\text { Geográfica }\end{array}$} & $100 \mathrm{~m}$ & $\mathrm{n}_{11}$ & $\mathrm{n}_{21}$ & $\mathrm{n}_{31}$ & $\ldots$ & $\mathrm{n}_{\mathrm{n} 1}$ \\
\hline & $200 \mathrm{~m}$ & $\mathrm{n}_{12}$ & $\mathrm{n}_{22}$ & $\mathrm{n}_{32}$ & $\ldots$ & $\mathrm{n}_{\mathrm{n} 2}$ \\
\hline & $300 \mathrm{~m}$ & $\mathrm{n}_{13}$ & $\mathrm{n}_{23}$ & $\mathrm{n}_{33}$ & $\ldots$ & $\mathrm{n}_{\mathrm{n} 3}$ \\
\hline & $\cdots$ & $\cdots$ & $\cdots$ & $\cdots$ & $\cdots$ & $\cdots$ \\
\hline & $100 \mathrm{n} \mathrm{m}$ & $\mathrm{n}_{1 \mathrm{n}}$ & $\mathrm{n}_{2 \mathrm{n}}$ & $\mathrm{n}_{3 \mathrm{n}}$ & $\ldots$ & $\mathrm{n}_{\mathrm{nn}}$ \\
\hline
\end{tabular}

Tabla 1. Tabla de contingencia de Knox (1964).

Una alternativa sería utilizar simulaciones de Monte Carlo para crear las distribuciones esperadas, las cuales no requieren esta condición (Besag \& Diggle, 1977). La simulación de Monte Carlo selecciona una muestra aleatoria de entre todas las permutaciones entre todos los puntos temporales disponibles, que luego se asocian con los puntos geográficos, cuyo orden se matiene constante. Se utiliza una muestra porque el número de permutaciones sería igual al factorial del número de incidencias el cual tiende al infinito al crecer el número de incidencias (ver tabla 2). 


\begin{tabular}{|r|r|}
\hline$n$ & $n !$ \\
\hline 0 & 1 \\
\hline 1 & 1 \\
\hline 2 & 2 \\
\hline 3 & 6 \\
\hline 4 & 24 \\
\hline 5 & 120 \\
\hline 6 & 720 \\
\hline 7 & 5040 \\
\hline 8 & 40320 \\
\hline 9 & 362880 \\
\hline 10 & 3628800 \\
\hline 15 & 1307674368000 \\
\hline 20 & 2432902008176640000 \\
\hline 25 & $1.19785717 \ldots \times 10^{100}$ \\
\hline 50 & $1.73368733 \ldots \times 10^{1,000}$ \\
\hline 70 & 15511210043330985984000000 \\
\hline 450 & $3.04140932 \ldots \times 10^{64}$ \\
\hline 3249 & $205703438 \ldots \times 10^{100,000}$ \\
\hline 25206 & \\
\hline &
\end{tabular}

Tabla 2. Factorial de n (n!) para varios valores de n.

Podemos entonces crear una tabla de contingencia como la anterior, donde se puedan comparar las frecuencias observadas con las generadas en las simulaciones. Si la frecuencia observada es mayor que la generada en el $99 \%$ de las permutaciones, nuestro resultado para esa casilla será significativo al nivel .01.

Utilizando este método, se ha demostrado que los robos de domicilio se aglomeran de forma espacio-temporal y de manera predecible (Johnson y Bowers, 2004; Johnson, Birks et al., 2007; Townsley et al., 2003). Por ejemplo, Johnson y Bowers Revista Española de Investigación Criminológica Artículo 1, Número 5 (2007) $\quad$ www.criminología.net 
(2004) mostraron cómo, después de un robo de domicilio, el riesgo de robo aumentaba de forma significativa en un radio de 400 metros durante el siguiente mes.

El objetivo de este estudio consiste en determinar si estas pautas espaciotemporales de robos de domicilio también existen en relación a robos de vehículos y sustracción de objetos en vehículos. Las cuestiones para las cuales se buscaron respuestas son las siguientes:

- ¿Existe una periodicidad general en el robo de vehículos y/o la sustracción de objetos en vehículo en relación a la hora del día, el día de la semana y/o la estación del año?

- ¿Es el riesgo de robo de vehículos y/o la sustracción de objetos en vehículo es contagioso (i.e. existe una aglomeración espacio-temporal?

- Si existe una aglomeración espacio-temporal, ¿a qué distancia y período de tiempo se eleva el riesgo de victimización?

- ¿Son las pautas espacio-temporales del robo de vehículos y del robo de objetos en vehículos distintos?

La identificación de estas pautas tiene un valor práctico obvio. Si el futuro delito se puede someter a simulación utilizando estos conocimientos, como se ha demostrado en el caso de robos de domicilio (Bowers et al., 2004), la policía podría actuar de una manera mucho más informada y así reducir y detectar el delito más eficazmente. 


\section{Métodos}

\subsection{Muestra}

Los datos analizados se obtuvieron de los cuerpos de policía de Derbyshire y Dorset (Reino Unido). Éstos consistían en denuncias de robos de vehículos y de sustracción de objetos en vehículos efectuadas durante el año 2005. En total, se hicieron 9,261 denuncias en Derbyshire y 5,747 en Dorset. Para cada una de estas denuncias, la siguiente información se hizo disponible:

- las fechas y horas que limitan la ventana temporal del robo (i.e. la última vez que se vio el vehículo antes del robo y cuándo se descubrió); ${ }^{\mathrm{i}}$

- la ubicación del robo (i.e. dirección); y

- las coordenadas geográficas del robo.

La exactitud de las coordenadas geográficas podría ser problemática, especialmente para este tipo de delito. La razón es que, en las bases de datos de la policía británica, las coordenadas normalmente se asignan automáticamente a partir de direcciones postales de residencias o negocios, mientras que muchos de estos delitos ocurren en aparcamientos o en la vía pública. Después de excluir las denuncias de robo que ocurrieron en aparcamientos ${ }^{\mathrm{ii}}$, tan sólo el 1,3\% de las denuncias en Derbyshire (112) y el $0,7 \%$ de las denuncias en Dorset (35), no contenían ninguna información sobre la ubicación del robo (por medio de coordenadas geográficas o dirección). Estas denuncias fueron eliminadas de la muestra. 
También se eliminaron de la muestra aquellas denuncias para las que no existía una dirección completa (i.e. localidad, vía y número o nombre del edificio o del negocio; aproximadamente el $40 \%$ de las denuncias en Derbyshire y el 35\% en Dorset), puesto que las coordenadas geográficas en estos casos son menos fiables. Por ejemplo, si sólo datos sobre la localidad y la vía son facilitados, las coordenadas asignadas se corresponden al punto medio de esta vía, lo que puede afectar los resultados, especialmente en casos de vías de mayor longitud.

Finalmente, las denuncias cuyas ventanas temporales excedían las 48 horas (aproximadamente el 10\%) también fueron eliminadas de la muestra, ya que la duración de esta ventana temporal afecta la precisión de las pautas temporales.

La muestra final resultó en 3,445 denuncias por sustracción de objetos en vehículos y 1,107 por robo de vehículos en Derbyshire y 2,444 denuncias por sustracción de objetos en vehículo y 366 por robo de vehículo en Dorset. Una inspección manual de una muestra aleatoria de estas denuncias (5\%) demostró que las coordenadas geográficas se habían asignado correctamente en más del 95\% de los casos. Por lo tanto, se concluyó que los datos de la muestra final eran apropiados para el análisis.

\subsection{Diseño y Análisis}

Los datos se sometieron a análisis estadísticos descriptivos para determinar las pautas temporales, espaciales y espacio-temporales. Las pautas espaciales fueron además evaluadas por 'análisis del vecino más próximo' (NNA, por sus siglas en inglés, 'Nearest Neighbour Analysis'). Para evaluar el significado estadístico de las pautas espacio-temporales se utilizaron tests de regresión múltiple y simulaciones de Monte Carlo. 


\section{Resultados}

\subsection{Análisis preliminar}

Puesto que las predicciones basadas en técnicas epidemiológicas sólo se hacen en relación a períodos temporales muy cortos, es importante establecer la rapidez de las fuerzas policiales en añadir los datos de denuncias a sus bases de datos. En Derbyshire, el $92 \%$ de las denuncias de robo de objetos en vehículo y el $94 \%$ de las denuncias de robos de vehículos son añadidos a la base de datos de la policía en menos de 24 horas. En el caso de Dorset, estas figuras son $92 \%$ y $89 \%$. Estos porcentajes son considerados satisfactorios.

\subsection{Pautas Temporales}

Como se puede observar en las figuras 1 y 2, los robos de vehículos se mantienen constantes a lo largo del año, mientras que las sustracciones de objetos en vehículos tienen períodos de más actividad, aunque éstos son diferentes en los dos condados.

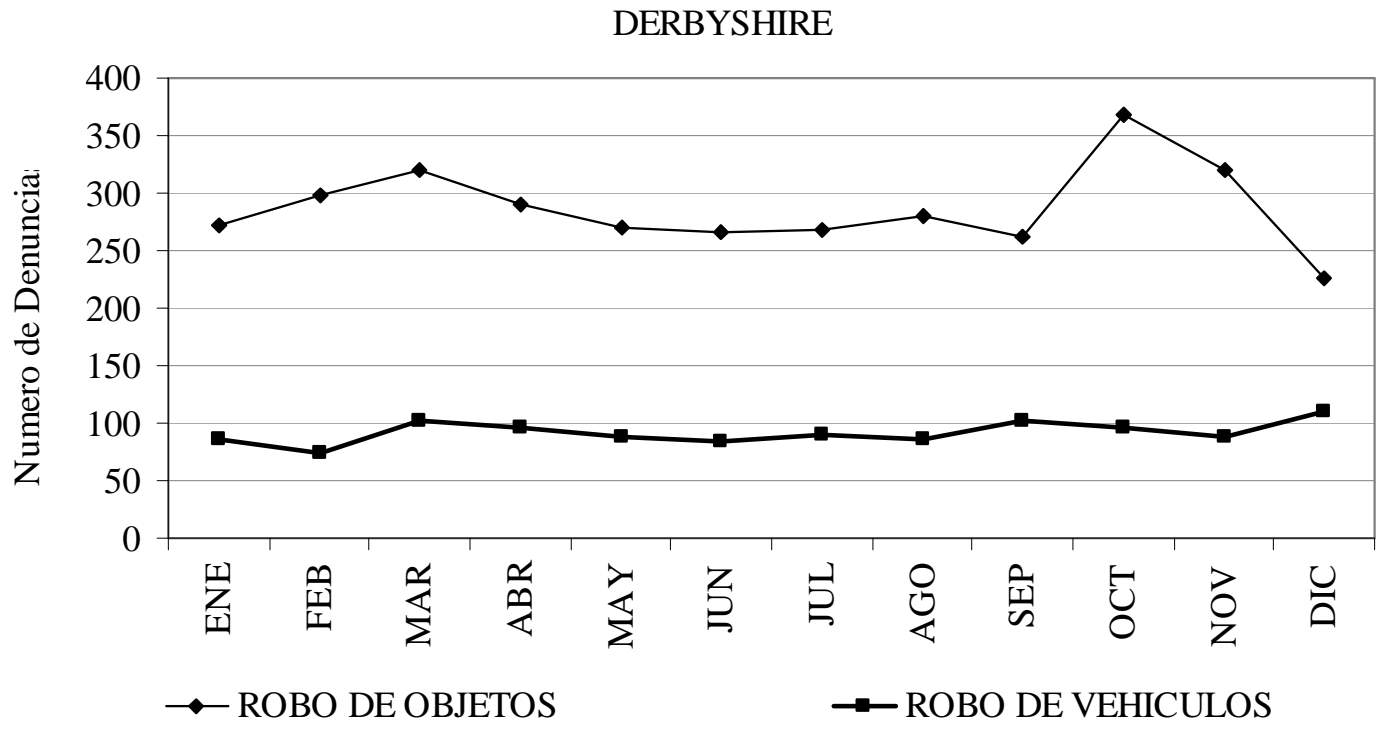

Figura 1. Pautas mensuales de los robos de vehículos y las sustracciones de objetos en vehículos en Derbyshire en el 2005. 


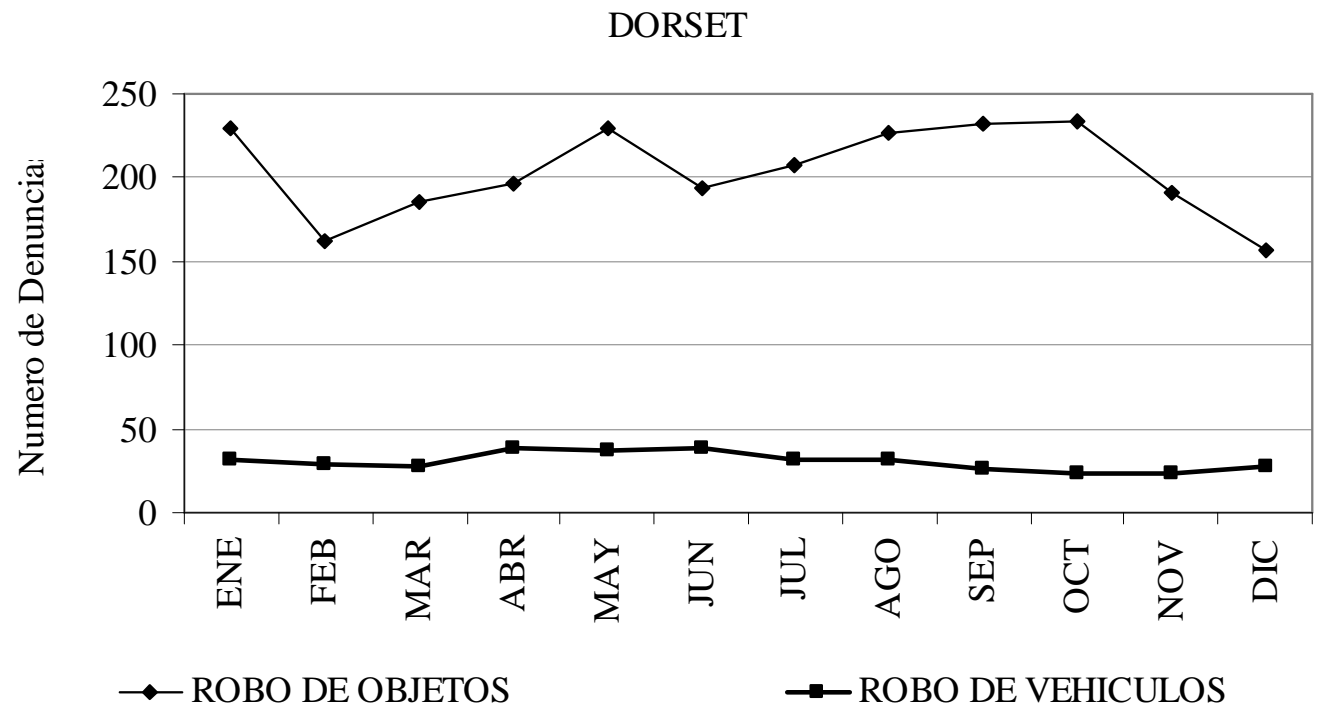

Figura 2. Pautas mensuales de los robos de vehículos y las sustracciones de objetos en vehículos en Dorset en el 2005.

\subsection{Pautas Espaciales}

Los mapas de zonas críticas realizados con estimaciones de densidad Kernel (KDE, por sus siglas en inglés ‘Kernel Density Estimation'; con particiones naturales) parecen indicar que los robos de vehículos y las sustracciones de objetos en vehículos se aglomeran en zonas especificas (figuras 3 y 4 ). 


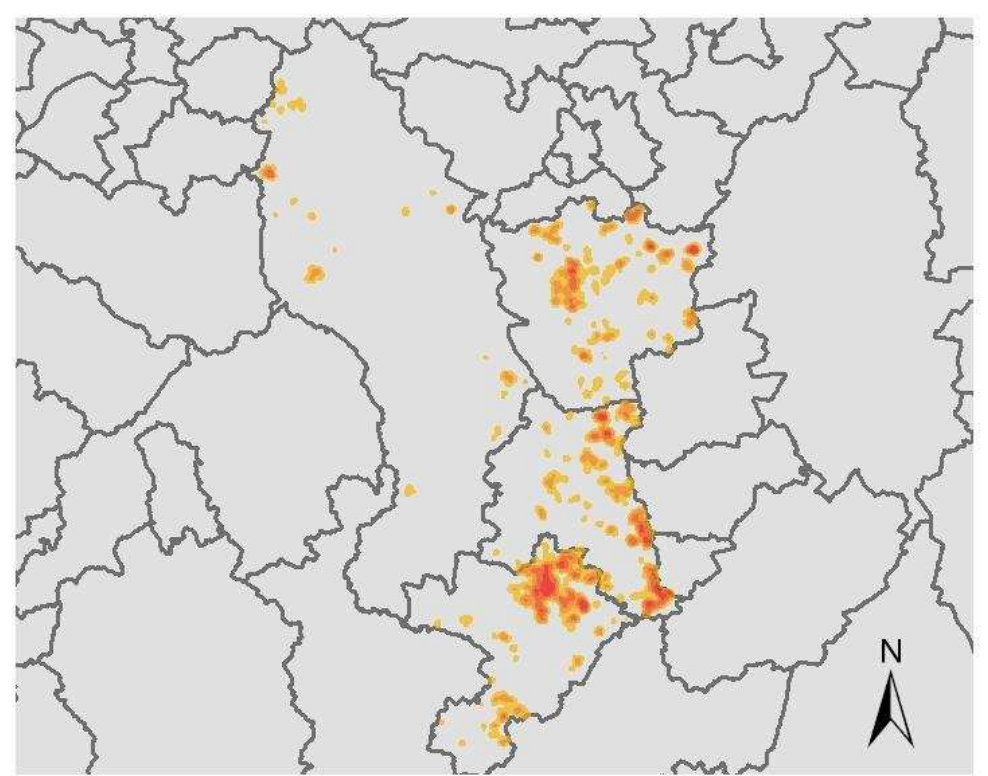

KDE for Theft from Motor Vehicle

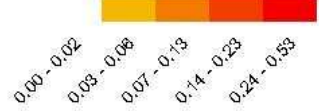

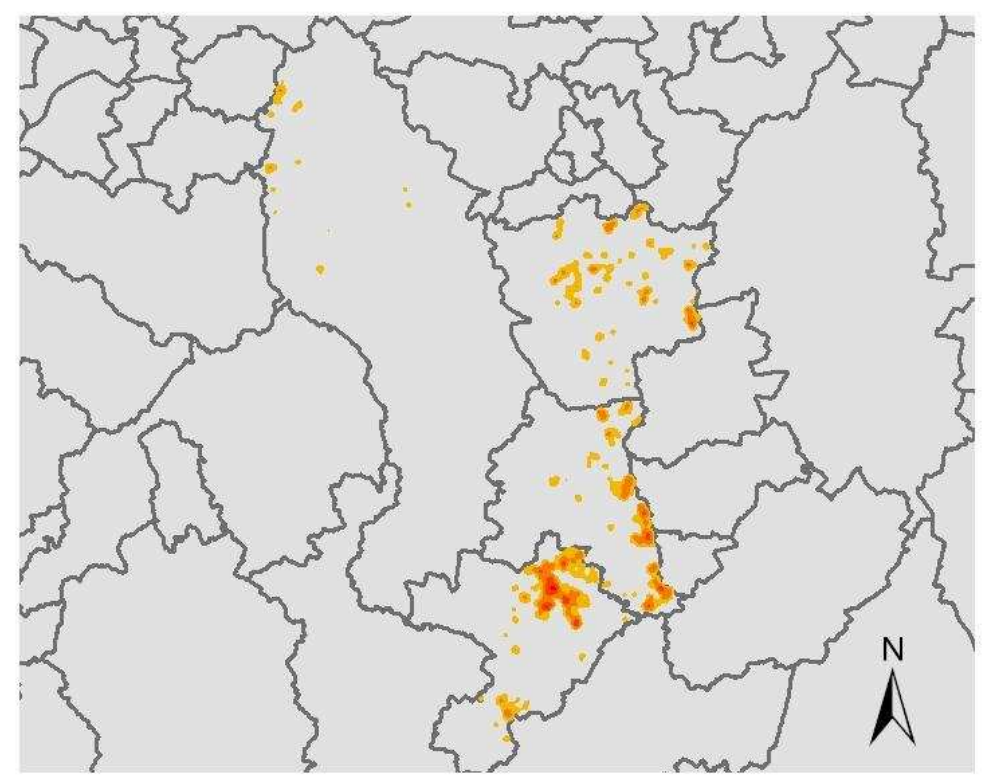

KDE for Theft of Motor Vehicle

$\begin{array}{lll}0 & 5 & 10\end{array}$ 20 Miles

Figura 3. Mapas de densidad Kernel de las denuncias por sustracción de objetos en vehículo (izquierda) y robo de vehículo (derecha) en Derbyshire (Reino Unido) en el 2005.

Revista Española de Investigación Criminológica

Artículo 1, Número 5 (2007) www.criminología.net 


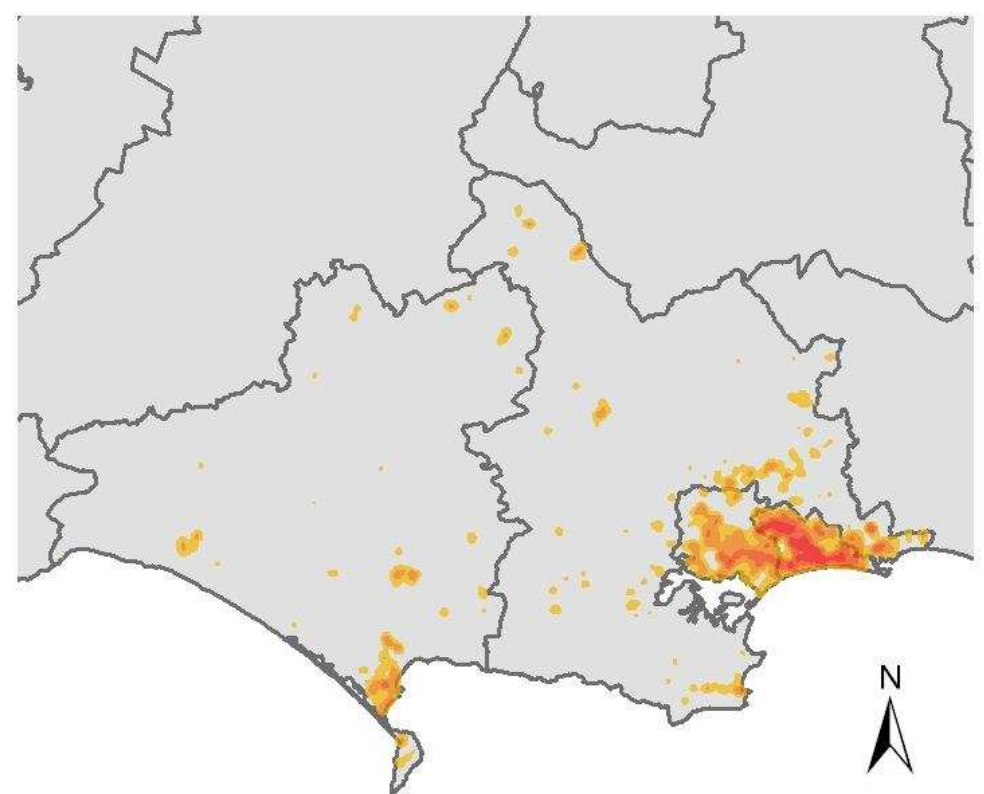

KDE for Theft from Motor Vehicle

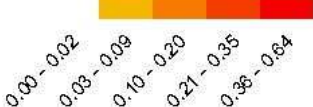

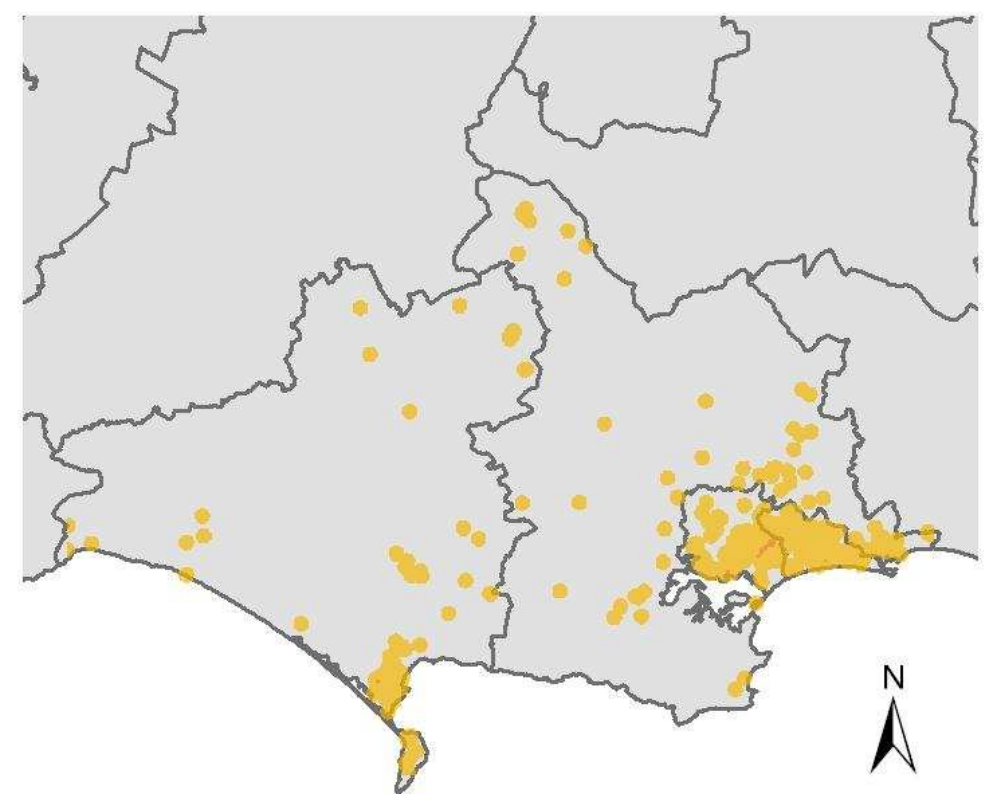

KDE for Theft of Motor Vehicle

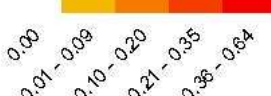
20 Miles

Figura 4. Mapas de densidad Kernel de las denuncias por sustracción de objetos en vehículo (izquierda) y robo de vehículo (derecha) en Dorset (Reino Unido) en el 2005.

Revista Española de Investigación Criminológica

Artículo 1, Número 5 (2007) www.criminología.net

ISSN: $1696-9219$ 
Análisis del vecino más próximo (NNA, por sus siglas en inglés 'Nearest Neighbour Analysis') revelaron que las diferencias entre las distancias medias entre cada robo y su vecino más próximo (i.e. el robo más cercano a éste) eran significativamente diferentes cuando éstas se compararon con las medias de una distribución aleatoria (Tabla 3).

\begin{tabular}{llrrr}
\hline & & Distancia Media (metros) & Valor Z & Probabilidad \\
\hline $\begin{array}{l}\text { Robo de } \\
\begin{array}{l}\text { Objetos en } \\
\text { Vehículo }\end{array}\end{array}$ & Derbyshire & 175 & -70.3 & $<.0001$ \\
\hline $\begin{array}{l}\text { Robo de } \\
\text { Vehículo }\end{array}$ & Derbyshire & 163 & -76.0 & $<.0001$ \\
& Dorset & 319 & -42.9 & $<.0001$ \\
& & 626 & -24.6 & $<.0001$ \\
\hline
\end{tabular}

Tabla 3. Resultados de los análisis del vecino más próximo de sustracción de (objetos en) vehículos en Derbyshire y Dorset (Reino Unido) en el 2005.

\subsection{Pautas Espacio-Temporales}

Una manera de explorar descriptivamente las pautas espacio-temporales consiste en calcular el porcentaje de robos ocurridos cerca de un antecedente en el espacio y el tiempo. Es decir, una vez que un robo ocurre, ¿cuántos robos ocurren en un radio de, por ejemplo, 400 metros y en menos de una semana?

Como se puede observar en las figuras 5 y 6, una vez que una sustracción de objetos en vehículo ocurre, del 20 al 30 por ciento de los otros robos suceden en menos de una semana y del 30 al 40 por ciento en menos de dos semanas. Esto indica una aglomeración espacio-temporal que se ajusta a una función logarítmica (calculada por medio de regresión múltiple), donde los robos que están cerca geográficamente suelen ocurrir en rápida sucesión, y es menos probable que se repitan mientras más tiempo pase del robo original. Sin embargo, sólo el 5\% de los robos ocurren en el mismo día, lo que da oportunidad a la policía de intervenir. 
En el caso de robo de vehículos, estas pautas no aparecen tan pronunciadas (figuras 7 y 8). En Dorset, las pautas se ajustan más a una función lineal que una logarítmica, que es lo que se esperaría si las pautas temporales y espaciales fueran totalmente independientes.

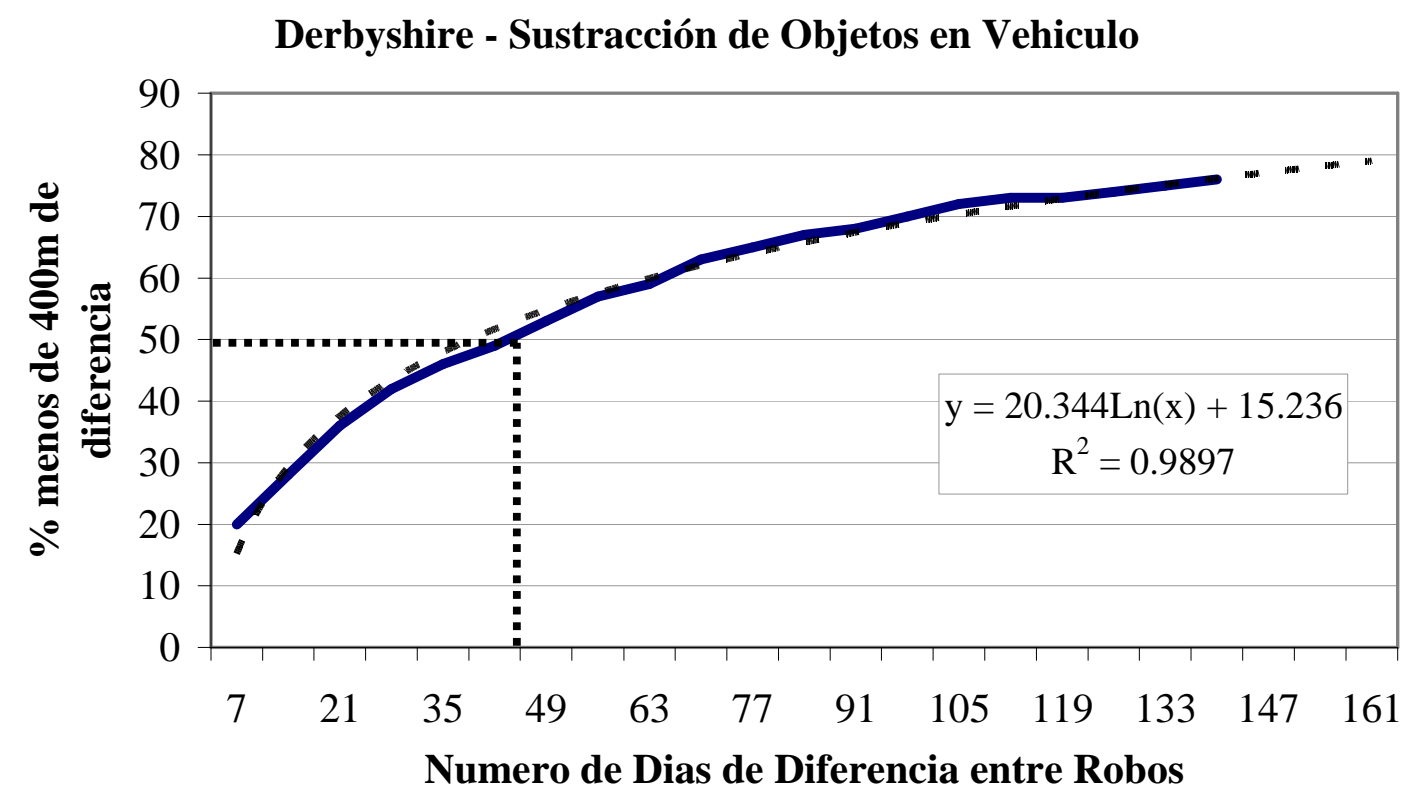

Figura 5. Porcentaje acumulativo de sustracción de objetos en vehículo en Derbyshire (Reino Unido) en el 2005 que ocurrieron en un radio de 400 metros del robo original. 


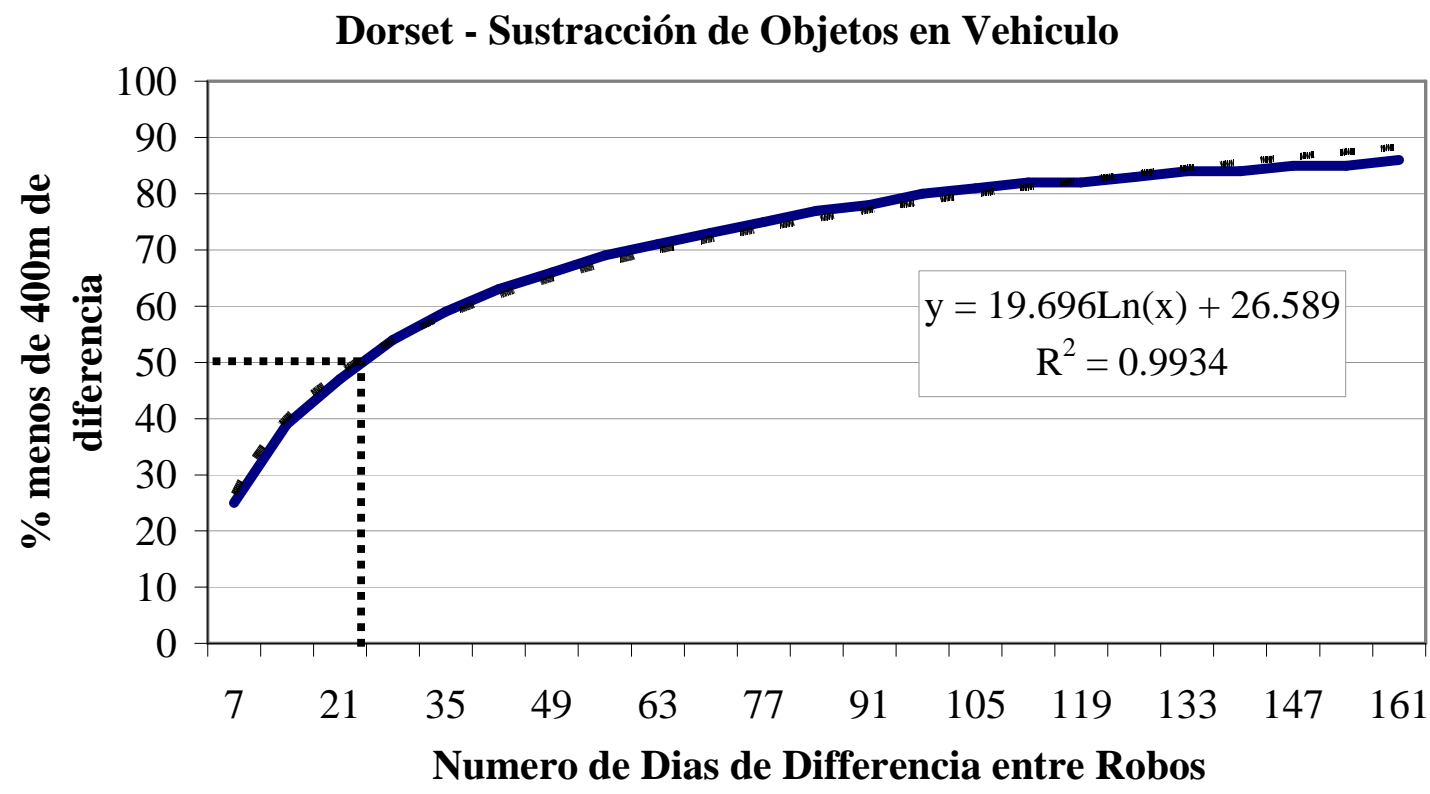

Figura 6. Porcentaje acumulativo de sustracción de objetos en vehículo en Dorset (Reino Unido) en el 2005 que ocurrieron en un radio de 400 metros del robo original.

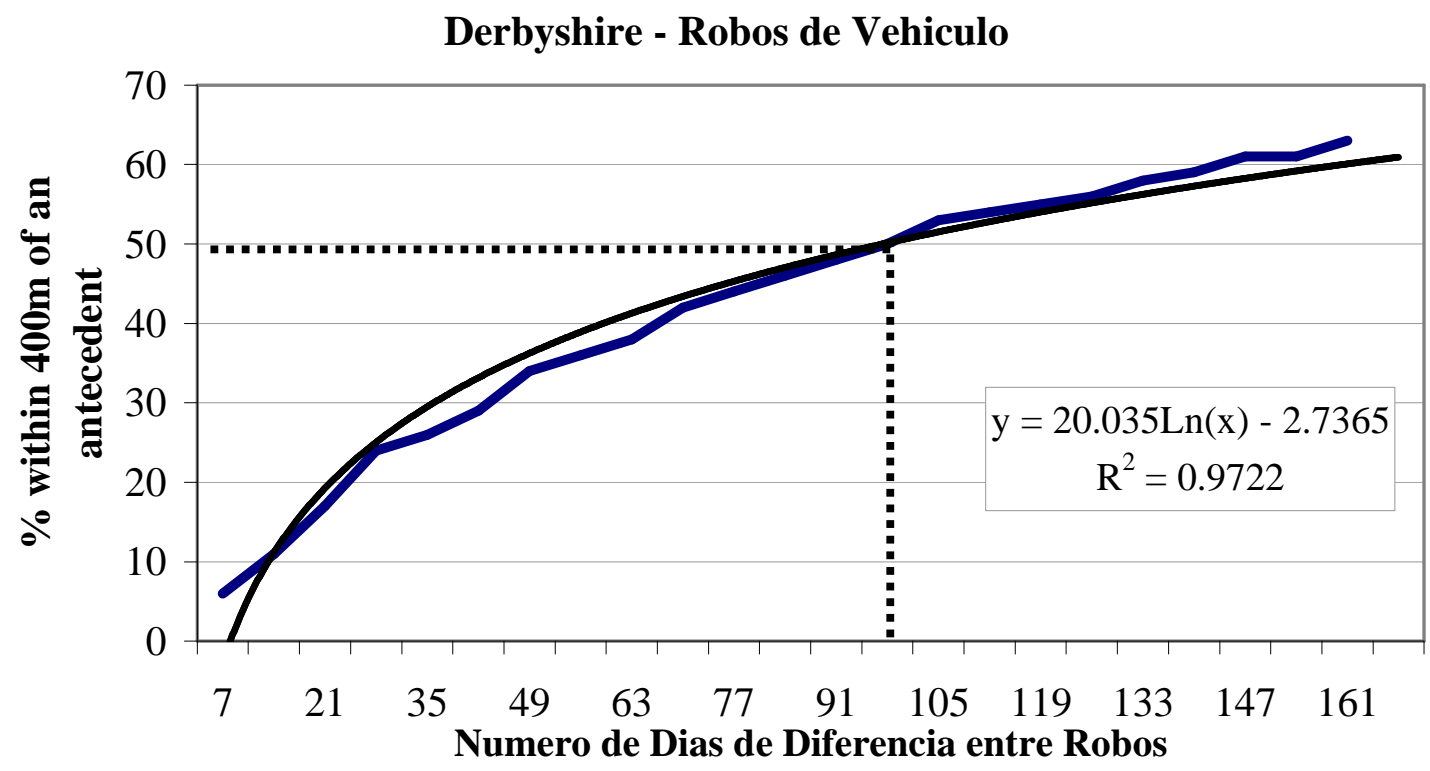

Figura 7. Porcentaje acumulativo de robos de vehículo en Derbyshire (Reino Unido) en el 2005 que ocurrieron en un radio de 400 metros del robo original. 


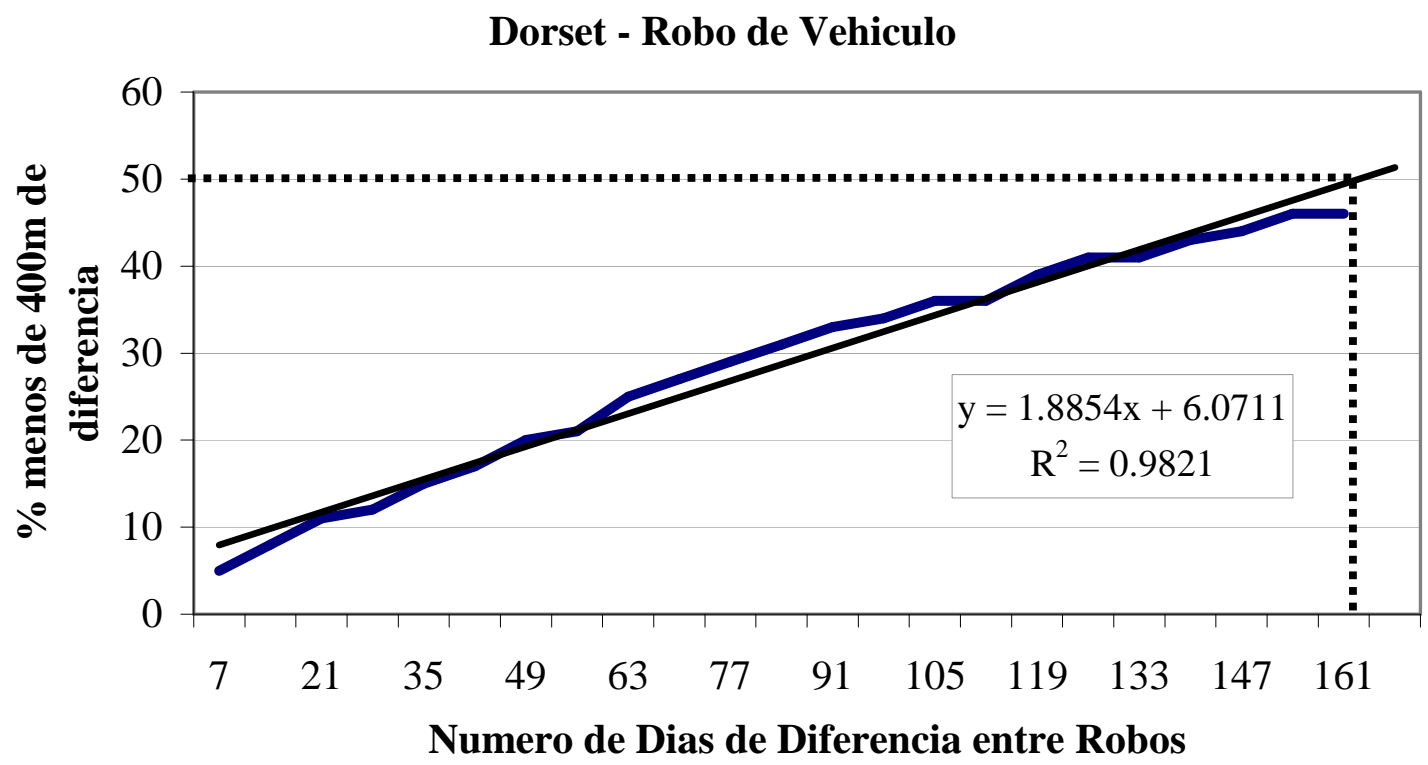

Figura 8. Porcentaje acumulativo de robos de vehículo en Dorset (Reino Unido) en el 2005 que ocurrieron en un radio de 400 metros del robo original.

\subsection{El Uso de Técnicas Epidemiológicas para Comprobar el Significado Estadístico de Pautas Espacio-Temporales}

Simulaciones de Monte Carlo se utilizaron para generar una muestra aleatoria de las posibles permutaciones en los datos, con el propósito de crear una distribución esperada. Una vez que esta distribución se comparó a los datos observados, los cocientes (i.e. observado sobre esperado) resultaron ser significativamente mayores en el caso de pares de sustracciones de objetos de vehículo más próximos temporal y geográficamente (figuras 9 y 10). Los robos de vehículo no mostraron las mismas pautas (figura 11; este análisis no se pudo realizar en Dorset por el reducido número de denuncias). 


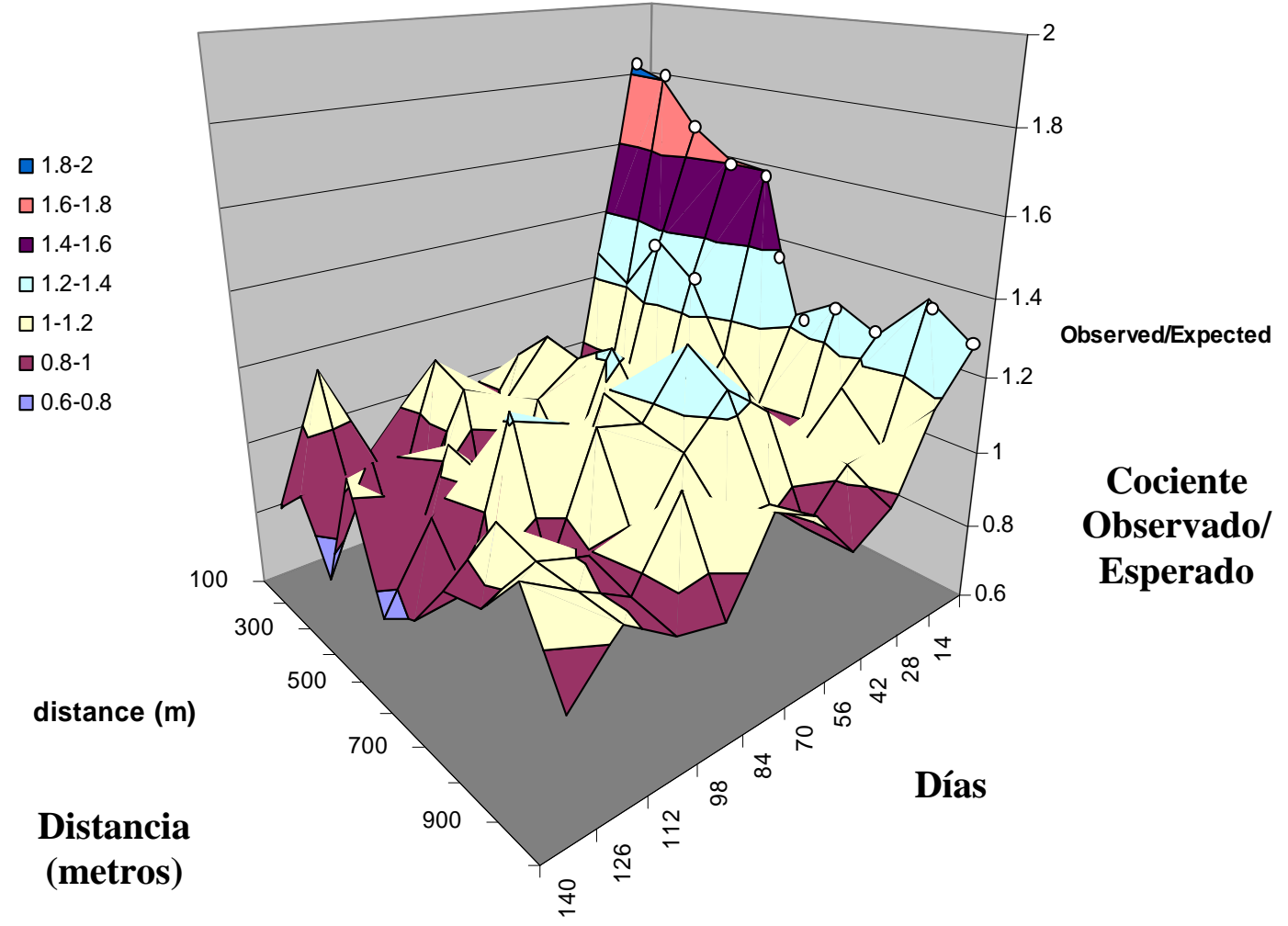

Figura 9. Superficie de los cocientes entre los números de pares de sustracciones de objetos en vehículos esperados y observados a distintas distancias y con distintos períodos de tiempo entre ellos, en la división C de Derby (Derbyshire, Reino Unido) en el 2005 (los puntos blancos indican los cocientes significativos estadísticamente al nivel .01). 
Q $13-15$

ㅁ.1.13

व09-1.1

$\square 0.7-09$

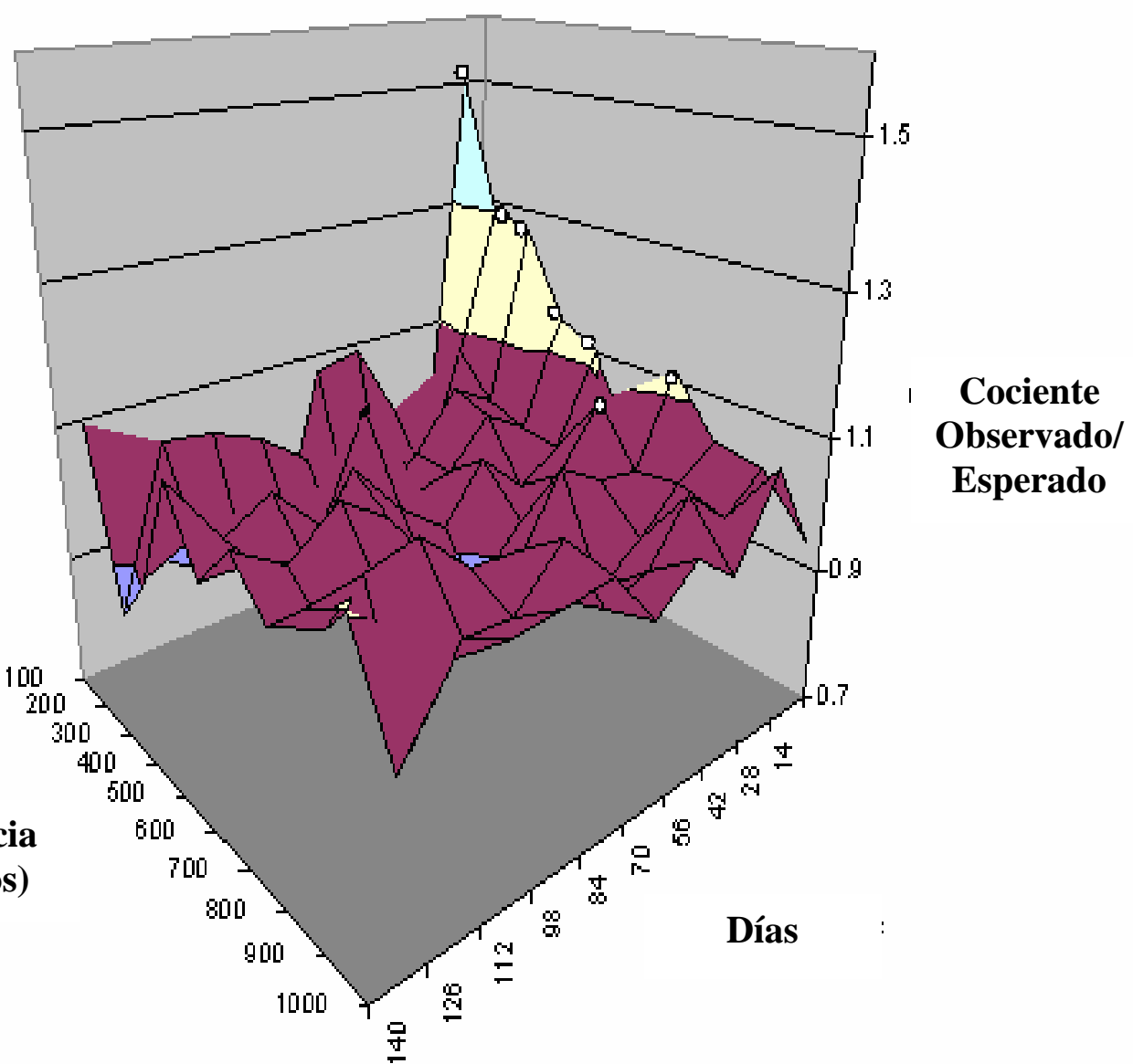

Figura 10. Superficie de los cocientes entre los números de pares de sustracciones de objetos en vehículos esperados y observados a distintas distancias y con distintos períodos de tiempo entre ellos, en Bornemouth (Dorset, Reino Unido) en el 2005 (los puntos blancos indican los cocientes significativos estadísticamente al nivel .01). 


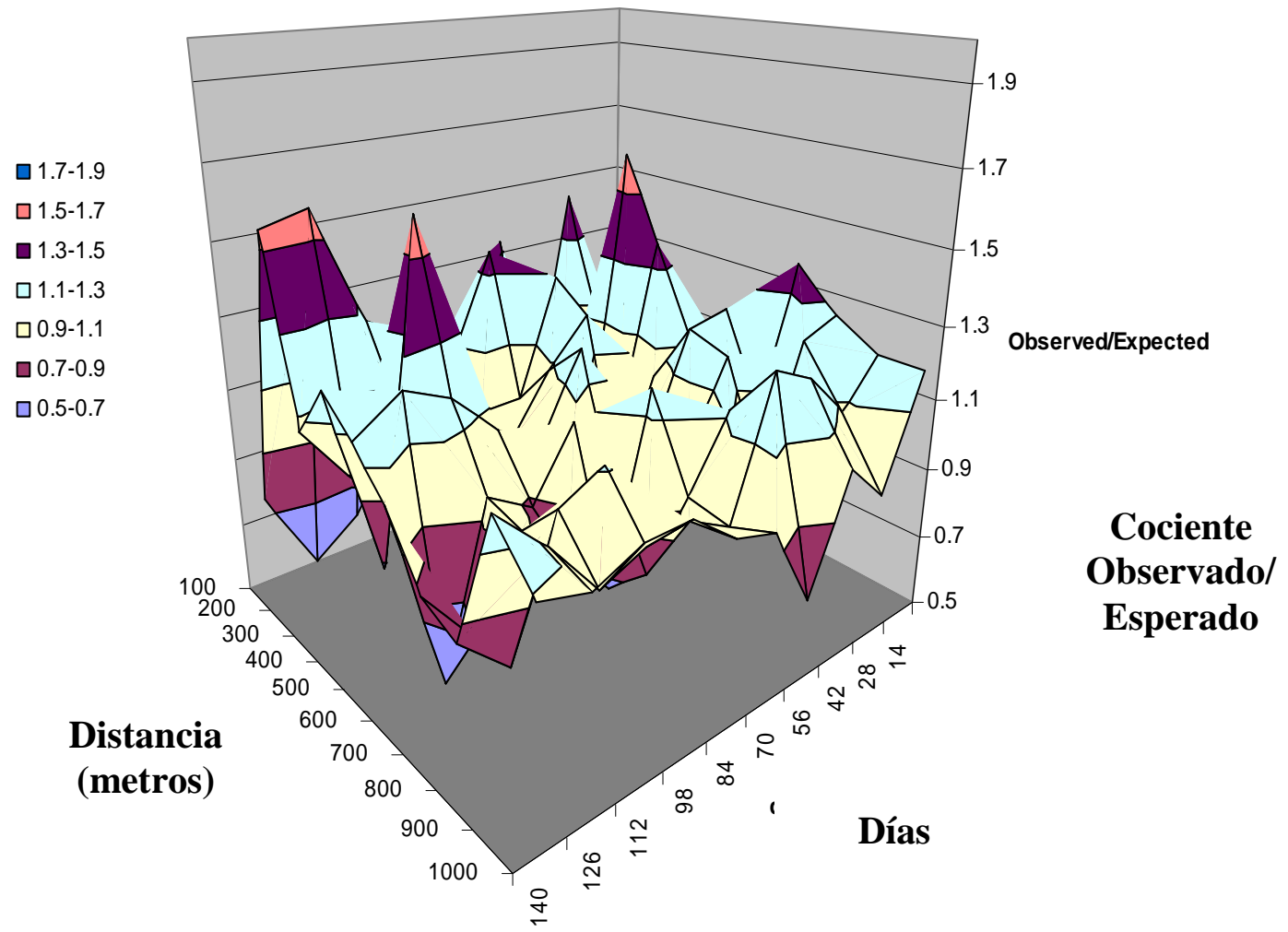

Figura 11. Superficie de los cocientes entre los números de pares de robos de vehículos esperados y observados a distintas distancias y con distintos períodos de tiempo entre ellos, en la división A de Derby (Derbyshire, Reino Unido) en el 2005 (los puntos blancos indican los cocientes significativos estadísticamente al nivel .01). 


\section{Discusión}

Los análisis presentados en este artículo han demostrado que, como en los robos de domicilio, existe una aglomeración espacio-temporal de sustracciones de objetos en vehículos; es decir, una vez que una sustracción ocurre, es más probable que otra ocurra a poca distancia geográfica y temporal de la original. En términos epidemiológicos, el riesgo de victimización en este caso es altamente contagioso.

Sin embargo, estas pautas no existen en el caso de robo de vehículos, lo que puede indicar que los delincuentes utilizan distintas "estrategias de caza" (por ejemplo, debido al mayor nivel de actividad requerido después de robar un vehículo).

Estos análisis se pueden ver limitados por la incorrecta o ambigua localización del lugar exacto del robo, especialmente en el caso de aparcamientos, los cuales fueron excluidos de nuestra muestra. A pesar de estas dificultades, los modelos observados son lo suficientemente fiables como para asumir que el trazado de mapas predictivos basados en estos conocimientos podría ser útil y contribuir a la prevención del delito y la detección y detención de delincuentes.

Estudios de seguimiento deben determinar si estas pautas son aplicables a otros tipos de delito (incluido el robo de vehículos y la sustracción de objetos en vehículo en aparcamientos) y también si hay interacción entre ellos, con el propósito de determinar la fiabilidad de estos modelos para el delito en general. Estudios cualitativos en los que los delincuentes expliquen sus estrategias de búsqueda e identificación de vehículos u otros objetos que robar también nos ayudarán a establecer la capacidad explicativa de los modelos. 


\section{Referencias}

Besag, J. \& Diggle, P.J. (1977). Simple Monte Carlo tests for spatial patterns. Applied Statistics, 26, 327-333.

Bowers, K.J.; Johnson, S.D. y Pease, K. (2004). Prospective hot-spotting: The future of crime mapping? The British Journal of Criminology, 44(5), 641-658.

Chainey, S. y Ratcliffe, J. (2005). GIS and Crime Mapping. London: Wiley.

Johnson, S.D. y Bowers, K.J. (2004). The burglary as cue to the future: The beginnings of prospective hot-spotting. European Journal of Criminology, 1(2), 237-255.

Johnson, S.D.; Birks, D.; Bowers, K.J.; McLaughlin, L. y Pease, K. (2007). Prospective Mapping Technical Report. Informe para el Ministerio del Interior Británico (Home Office).

Johnson, S.D.; Bernasco, W.; Bowers, K.J.; Elffers, H.; Ratcliffe, J.; Rengert, G. y Townsley, M.T. (2007). Near repeats: A cross national assessment of residential burglary. Journal of Quantitative Criminology, en prensa.

Knox, G. (1964). The detection of space-time interactions. Applied Statistics, 13, 25-29.

Pease, K. (1998). Repeat victimisation: Taking stock. Crime Detection and Prevention Series Paper 90. London: Home Office. 


\section{Currículo de los Autores}

Lucía Summers es licenciada en Psicología por la Universidad de Sussex (Reino Unido), y también ha obtenido Másters en Psicología Criminal Aplicada (Universidad de Leicester, Reino Unido) y en Métodos de Investigación para Psicología (University College London, Londres, Reino Unido). Actualmente, Summers está haciendo su doctorado en colaboración con la Policía Metropolitana de Londres, Reino Unido, el cual está enfocado al estudio situacional del homicidio. Otros proyectos de investigación en los que Lucía ha trabajado han abordado temas tales como el delito relacionado con el uso de drogas, el delito contra locales comerciales y la confiscación de bienes provenientes de actividades delictivas.

Dr. Shane D. Johnson es Doctor en Criminología y profesor en el UCL Jill Dando Institute (Londres, Reino Unido). Johnson lleva más de diez años trabajando en los campos de criminología y psicología criminal, durante los cuales ha publicado más de 40 artículos y finalizado proyectos de investigación en el Reino Unido para el Ministerio del Interior (Home Office), la policía y el Ministerio de Medio Ambiente (Department for the Environment and Rural Affairs o DEFRA). También ha recibido numerosas becas de investigación, de organizaciones tan importantes como la Academia Británica (British Academy) y el Consejo de Investigación de Arte y Humanidades (Arts and Humanities Research Council).

Prof. Ken Pease es profesor visitante del UCL Jill Dando Institute y de la Universidad de Loughborough (Reino Unido). Pease ha publicado más de 200 libros, monografías de investigación y artículos, y recientemente le fue otorgado el O.B.E. (Order of the British Empire u Orden del Imperio Británico) por sus servicios en el campo de la prevención del delito. 


\section{Agradecimientos}

Los autores desean agradecer a las fuerzas policiales de Derbyshire y Dorset en el Reino Unido por facilitarles el acceso a los datos, en especial a Derek Johnson, de la policía de Dorset. También desean agradecerle a Juan Angel Anta sus numerosas revisiones y a la Home Office de Londres por patrocinar este proyecto.

\section{Notas}

\footnotetext{
i Cuando un robo de vehículo o una sustracción de objetos en vehículo es denunciado a la policía, normalmente no es posible saber con exactitud cuándo ocurrió el robo. De esta forma, la policía toma nota de una ventana temporal desde que el vehículo fue visto la última vez antes del robo hasta que el robo fue descubierto.

ii Los datos referentes a robos que ocurrieron en aparcamientos (574 en Derbyshire y 934 en Dorset) fueron eliminados de la muestra debido a las diferencias contextuales de este tipo de incidente. Por ejemplo, el acceso a determinados aparcamientos está restringido a días y/u horas específicas, lo que puede afectar los resultados. Además de esto, los robos en aparcamientos son difíciles de codificar geográficamente.
} 